\title{
Physical Properties and Characteristics of III-V Lasers on Silicon
}

\author{
Graham William Read, Igor Pavlovich Marko, Nadir Hossain, Member, IEEE, \\ and Stephen John Sweeney, Senior Member, IEEE
}

(Invited Paper)

\begin{abstract}
The development of laser technology based on silicon continues to be of key importance for the advancement of electronic-photonic integration offering the potential for high data rates and reduced energy consumption. Progress was initially hindered due to the inherent indirect band gap of silicon. However, there has been considerable progress in developing ways of incorporating high gain III-V based direct band gap materials onto silicon, bringing about the advantages of both materials. In this paper, we introduce the need for lasers on silicon and review some of the main approaches for the integration of III-V active regions, including direct epitaxial growth, hybrid integration, defect blocking layers and quantum dots. We then discuss the roles of different carrier recombination processes on the performance of devices formed using both wafer fusion and direct epitaxial approaches.
\end{abstract}

Index Terms-Carrier recombination processes, efficiency, photonic integration, silicon photonics, temperature sensitivity, III-V epitaxy.

\section{INTRODUCTION}

D EMAND for both data processing power as well as internet access and versatile online content are growing exponentially, from both developed and increasingly from developing nations [1]. As a result the energy consumption attributed to computing, data centers and communications is increasing substantially. It is predicted that at current growth rates the energy consumption associated with the internet alone could theoretically reach between $22 \%$ and $100 \%$ of the global electricity supply by 2023 , assuming annual reductions in power consumption per $\mathrm{Gbs}^{-1}$ of throughput of $15 \%$ (optimistic) or $0 \%$ (pessimistic), respectively [2]. Since demand will not decrease, the efficiency and flexibility of the hardware and systems at the heart of the network infrastructure must be increased.

To keep pace with faster processors, demand for bandwidth across servers, boards and on-chip must increase to prevent bottlenecking. Electrical data transfer reaches its practical limit

Manuscript received February 6, 2015; revised April 15, 2015; accepted April 18,2015 . This work was supported in part by the Engineering and Physical Sciences Research Council, U.K., under Grant EP/H005587/1. Details of the data and how to request access are available from the University of Surrey publications repository at http://epubs.surrey.ac.uk/807592/.

G. W. Read, I. P. Marko, and S. J. Sweeney are with the Advanced Technology Institute and Department of Physics, University of Surrey, Surrey GU2 7XH, U.K. (e-mail: s.sweeney@surrey.ac.uk).

N. Hossain was with the Advanced Technology Institute and Department of Physics, University of Surrey, Surrey GU2 7XH, U.K. He is now with the INRS-EMT, Canada.

Color versions of one or more of the figures in this paper are available online at http://ieeexplore.ieee.org.

Digital Object Identifier 10.1109/JSTQE.2015.2424923 at a bandwidth distance product of $\sim 100 \mathrm{Gbs}^{-1} \mathrm{~m}$ [3] due to the fundamental physical limitations of electrical interconnects [4]. Thus, there are two key issues to address, both of which can be resolved via optoelectronic integration on silicon. Optical data transfer, having already been adopted over scales from 1000s of km down to a few metres offers far greater bandwidth, through smaller interconnects than its electrical counterpart. Energy loss through resistive heating is eliminated and, with cooling and data transfer accounting for the bulk of data center energy consumption, the overall energy demands of a photonic system would be vastly reduced [5].

The main components required for such a system have mostly been realized in a viable form, including modulators operating up to $50 \mathrm{~Gb} / \mathrm{s}$ [6], Ge/Si photodetectors, filters, waveguides, switches and even micro-electro-mechanical systems, enabling the dynamic control of optical signals [7]. The main component yet to be developed sufficiently for commercialization is a suitable light source on silicon.

The aim of this paper is to review some of the main current approaches to develop silicon-based lasers utilizing III-V based gain media, emphasizing current progress and the remaining key challenges. The paper goes on to discuss in further detail two of the primary approaches used to date, namely wafer fusion and direct epitaxial growth, in terms of the physical properties and performance characteristics of the devices and the prospects for future development.

\section{REVIEW OF SiLICON COMPATIBLE LASERS}

The development of efficient and temperature stable lasers on $\mathrm{Si}$ is therefore essential to meeting the future communication and computing demands. The lasers must be compatible with CMOS processes in order to be integrated in a commercially viable manner with $\mathrm{Si}$-micro-electronics. It is well known that bulk $\mathrm{Si}$ is not a suitable laser active region due to its indirect bandgap meaning that gain is intrinsically weak and nonradiative processes are problematic [8]. This has led to many and varied and often complicated techniques to develop a laser on Si. Many approaches to improving the light emission properties of Si have been investigated. Si nanocrystals [9], porous Si [10], Si nano-pillars [11] and Si-insulator superlattices all make use of the fact that low-dimensional $\mathrm{Si}$ is found to emit light at increased efficiency at room temperature. Furthermore, exploitation of stimulated Raman scattering has been used to produce an optically pumped continuous wave $(\mathrm{CW})$ laser operating at room temperature [12]. Common to all these approaches, 
however, is a strong free carrier absorption lowering the gain [13], saturation of the luminescence at high power operation due largely to Auger recombination [14] and the strong dependence of Si feature size on emission energy leading to inhomogeneous broadening and optical loss [15]. As a result, efficient electrical injection lasing remains elusive.

An alternative is the integration of the proven, efficient, commercialised III-V active materials with the excellent waveguiding and electrical properties of $\mathrm{Si}$. The inherent problem facing the epitaxial growth of III-Vs on Si is the large lattice and thermal expansion coefficient mismatch between $\mathrm{Si}$ and common III-Vs as well as the fact that they form a polar/non-polar interface [16]. These factors induce strain in epitaxially deposited layers, which is accommodated by the formation of large densities of defects during growth, rendering efficiency and lifetime impractically low.

The various approaches to the integration of III-Vs on Si can be separated into two main categories. The first one is hybrid integration through the fusion of two independently grown wafers, one III-V based and the other silicon-based. By separating the growth of the two material systems, an almost defect free III-V wafer can be formed and any defects generated during fusion confined to the local vicinity of the bonding interface. Several approaches have been demonstrated and investigated with varying degrees of success including adhesive bonding [17], anodic bonding [18], self-aligned solder bumps [19], transfer printing [20] and low temperature oxygen plasma assisted flip chip wafer bonding [21]. For the most part these processes are based on standard CMOS chip-bonding procedures and as such are generally reliable. However, impeded conduction of heat generated within the III-V region to the substrate via the bonding region can lead to elevated internal temperatures and as a result reduced lifetime.

The second approach is the direct epitaxial growth of III-V material onto $\mathrm{Si}$. In order to achieve epitaxial growth either the strain or defects generated during growth must be compensated for. An approach accommodating the lattice mismatch is to develop new materials that more closely match the structural properties of Si. Defect free growth on $\mathrm{Si}$ of the active material GaNAsP along with strain compensating barrier and $\mathrm{SCH}$ materials BGaAsP and $\mathrm{BGaP}$ developed at Philipps-University, Marburg, Germany, has been demonstrated and is discussed in further detail below [22].

In order to use a proven and well optimised material system the lattice constant mismatch and resultant strain must be accommodated. However, the active region and injection path can be isolated from the defects through precise strain management, which causes the bending and subsequent annihilation of threading dislocations. Several approaches have been demonstrated, including III-Sb buffer layers [23], strained Stranski-Krastanow quantum dot layers [24], graded Si-Ge buffer layers [25] and strained super-lattices (SSL) [26].

While optoelectronic integration for communications and computing applications has been the main driver for the integration of III-Vs on $\mathrm{Si}$, it should be noted that further applications of interest are under development. For example, the use of inexpensive Si substrates for GaN LEDs rather than the more typi- cal sapphire or SiC. Solid state lighting, although more energy efficient than even the best energy efficient compact fluorescent lamps, is relatively expensive for widespread household adoption. Currently GaN LED wafers are produced with a diameter of only 2 or 3 in due to the difficulty of growing sapphire or $\mathrm{SiC}$ wafers of sufficient crystalline quality on a larger scale. The use of 6 in wafers is found to increase device yield by $44 \%$ for an equivalent amount of material, greatly reducing cost [27]. Si, being one of the most abundant elements on Earth and already in mass production is an inherently cheaper substrate material to start with. GaN/Si LEDs have not only been realised but are even approaching the performance of those on more standard substrates [27] and have recently been commercialised through Plessey Semiconductors in the UK [28]. Thus it is clear that the integration of III-V materials on silicon substrates has many important applications in photonics.

The remainder of the paper focuses on the optical communications applications of III-V lasers on silicon. In the following sections the current state of the field of III-V integration on $\mathrm{Si}$ is summarized along with further detail of several promising approaches that we have studied in collaboration with growth partners.

\section{HYBRID INTEGRATION APPROACH}

Hybrid integration has some advantages over direct epitaxial growth on $\mathrm{Si}$ and has led to rapid development in this field. Since mature material systems are integrated onto $\mathrm{Si}$, relatively little work is required in terms of optimising the structure of the III-V section. The main differences in the III-V sections of varying approaches relate to the region in which the optical field is confined. When confined to the III-V section, microdisks give a strong optical confinement factor and therefore large gain. These are generally defined through reactive ion etching of the III-V post bonding to the Si substrate. The use of micro-disks allows small form factor, beneficial for minimising overall package dimensions. However, through this approach the lasing wavelength is defined by the micro-disk diameter and is therefore somewhat problematic to target exact wavelengths. A 7.5- $\mu \mathrm{m}$ diameter micro-disk laser has been demonstrated with a threshold current of only $0.33 \mathrm{~mA}$. However, with injection of $3.8 \mathrm{~mA}$ (more than 10 times threshold, limited by thermal rollover) only $\sim 20 \mu \mathrm{W}$ of optical power is coupled into the waveguide [29].

An alternative approach is to design structures in which the optical field from the III-V layer is strongly coupled to the SOI waveguide, where precisely defined wavelength selective features such as a DFB grating can be incorporated using standard processing techniques. In this case the optical mode is evanescently coupled to a III-V mesa structure yielding a lower optical confinement factor and gain. The geometry of this approach does however yield larger form factor in order to maximise optical mode overlap and therefore yields larger threshold currents. Threshold currents of $\sim 20-30 \mathrm{~mA}$ have been demonstrated at which optical powers of $\sim 1 \mathrm{~mW}$ were achieved at $1.6 \mu \mathrm{m}$ [30]. More recently, longer wavelength, 2.0- $\mu \mathrm{m}$ lasers have been demonstrated, heterogeneously integrated on silicon 


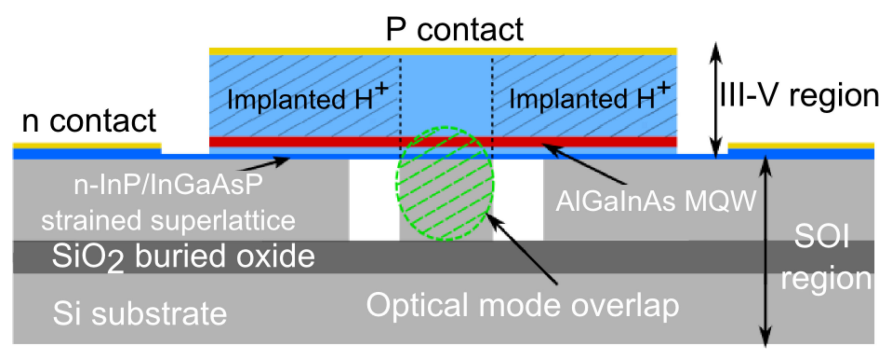

Fig. 1. Device structure of the hybrid AlGaInAs/InP silicon-evanescent laser.

employing molecular wafer bonding of InP to Si. These hybrid silicon lasers operated up to $35^{\circ} \mathrm{C}$ and emitted up to $4.2 \mathrm{~mW}$ of single-facet $\mathrm{CW}$ power at room temperature [31]. The choice of micro-disk or linear cavities requires balancing form factor and threshold current against optical power and ease of tuning.

The bonding process may take place in a single step, in which two wafers are bonded, with the III-V processing taking place post fusion. Alternatively individual dies may be bonded singularly or in batches. Use of solder bumps or epoxy, for example, requires bonding of individual dies, which by nature, makes the process comparatively slow and expensive compared with full wafer bonding. However, less III-V material is consumed in doing so and costs can be incurred through processing time or material consumption in either case. For wafer bonding there are two main approaches, direct surface adhesion using Van der Waals forces or adhesive bonding. In the case of the former a strong direct bond is formed but surfaces must be near atomically smooth $(\sim 0.5 \mathrm{~nm}$ tolerance) and totally free of contamination. Adhesive bonding on the other hand has far more relaxed tolerances, and provided that a suitable adhesive is used (such as benzocyclobutene- $\mathrm{BCB}$ ), strong, low optical loss and thermally stable bonds can be formed.

In this section we discuss measurements on hybrid siliconevanescent lasers developed at UCSB by the research group of J. Bowers. A cross-sectional diagram indicating the key components of the hybrid laser structure is presented in Fig. 1 for reference. These devices bond an AlGaInAs/InP III-V mesa to a pre-patterned SOI substrate in which the optical mode is confined in the SOI waveguide and evanescently coupled to the active region. The III-V mesa is defined post bonding with a current channel defined through proton implantation. A strained superlattice defect-blocking layer is included in the n-InP region in order to inhibit the propagation of threading dislocations, formed during bonding towards the active region. A DFB grating structure is defined in the pre-patterned SOI ridge waveguide through e-beam lithography pre-bonding yielding a temperature independent and narrow line width emission. Detailed information about the device fabrication process can be found in [32].

Due to the stringent tolerances on surface quality for direct wafer bonding, a multi-step surface preparation process is used to achieve high quality, homogenous bonding. Chemical surface treatment is followed by oxygen plasma exposure in order to remove all traces of water and organics and the wafers are brought together in situ within the RIE chamber. A pressure of $1.5 \mathrm{MPa}$ is applied for 12 hours at an annealing temperature of $300^{\circ} \mathrm{C}$. Full details of the bonding process can be found elsewhere [21].



Fig. 2. Electrical injection LI plots as a function of temperature with inset lasing spectra.

The inclusion of several periods of SSL in the III-V below the active region was found through TEM studies to effectively block the propagation of threading dislocations formed during wafer fusion into the active region. This was further backed by photoluminescence showing a seven fold increase in emission intensity after the inclusion of the SSL.

Devices were found to lase under electrical injection up to $60^{\circ} \mathrm{C}$, with an ambient temperature threshold current density of $\sim 4.5 \mathrm{kAcm}^{-2}$ (Fig. 2). Device yields in excess of $95 \%$ have been reported indicating uniform bonding quality across wafers. Furthermore the devices are found to be reliable with no significant degradation of threshold current density after $\sim 5000$ hours of $\mathrm{CW}$ operation at $70^{\circ} \mathrm{C}[30]$.

Emission is found to exhibit a narrow line width (see the insert in Fig. 2), resulting from the strong wavelength selectivity of the DFB grating. The threshold current increases and slope efficiency decreases rapidly with temperature suggesting the presence of temperature dependent loss mechanisms such as Auger recombination which has previously been reported in our earlier work on AlGaInAs active materials [33].

In order to develop a better understanding of the carrier recombination processes occurring in the devices, spontaneous emission was collected via an optical fibre placed in contact with the substrate of the device [34]. It can be seen from Fig. 3 that the pure spontaneous emission (after removal of scattered stimulated emission) does not pin above threshold. This is problematic, since if the carrier density is continuing to rise above threshold then mechanisms with a stronger carrier density dependence, such as Auger recombination and carrier leakage (see eq. (1)) will rapidly dominate recombination and can start to impact the slope efficiency.

The spontaneous emission, which is determined by the $B n^{2}$ term in eq.(1), can be used for analysis by considering the different current paths flowing in the devices. If the injection current is formed of defect, radiative, Auger and leakage components, it can be expressed using the equation below

$$
I=A n+B n^{2}+C n^{3}+I_{\text {leak }}
$$




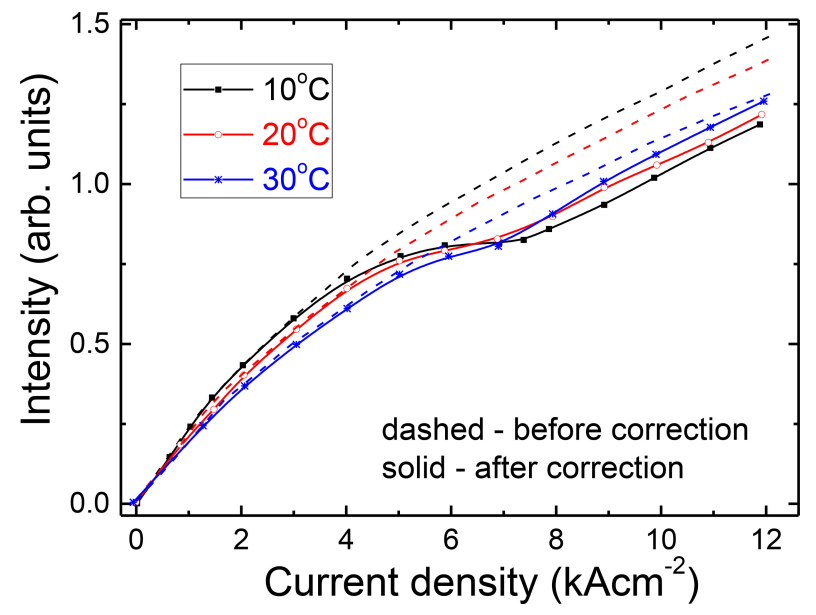

Fig. 3. Integrated spontaneous emission collected from the substrate before (dashed curves) and after correction (solid curves with symbols) by taking into account scattered stimulated emission.

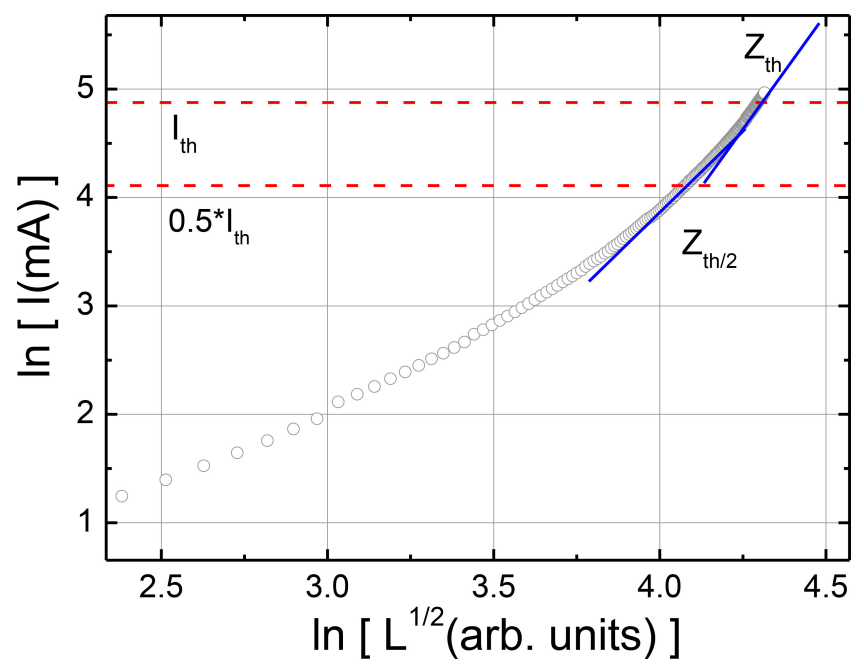

Fig. 4. $\quad \ln (\mathrm{I}) \mathrm{vs} \ln \left(\mathrm{L}^{1 / 2}\right)$, the gradient of which is taken to be " $\mathrm{Z}$ ". The dotted red lines represents the threshold and half threshold currents and the blue tangents the gradients ( $\mathrm{Z}$ values) at those currents.

where $A, B$ and $C$ are the defect, radiative and Auger recombination coefficients, respectively, and $n$ is the carrier density assuming that the electron and hole densities are equal.

The so-called $\mathrm{Z}$ analysis [34], may then be used to determine which of these mechanisms dominates the devices under investigation. $\mathrm{Z}$ is defined by plotting $\ln (I)$ vs $\ln \left(L^{1 / 2}\right)$, where $L$ is the integrated pure spontaneous emission collected perpendicular to the laser cavity [34]. Thus, from eq. 1 the gradient of this plot represents the power law dependence of the injection current on carrier density as shown in Fig. 4. By repeating this as a function of temperature it is possible to determine the extent to which different current paths dominate at different operating temperatures. In Fig. 5 we plot the temperature dependence of the $\mathrm{Z}$ value extracted close to the threshold current and also at approximately $1 / 2 J_{\text {th }}$ as a function of temperature.

An increasing $\mathrm{Z}$ value at threshold as a function of temperature, exceeding a value of 3 was determined (see Fig. 5.). From eq. (1), $Z=3$ is indicative of Auger recombination, which

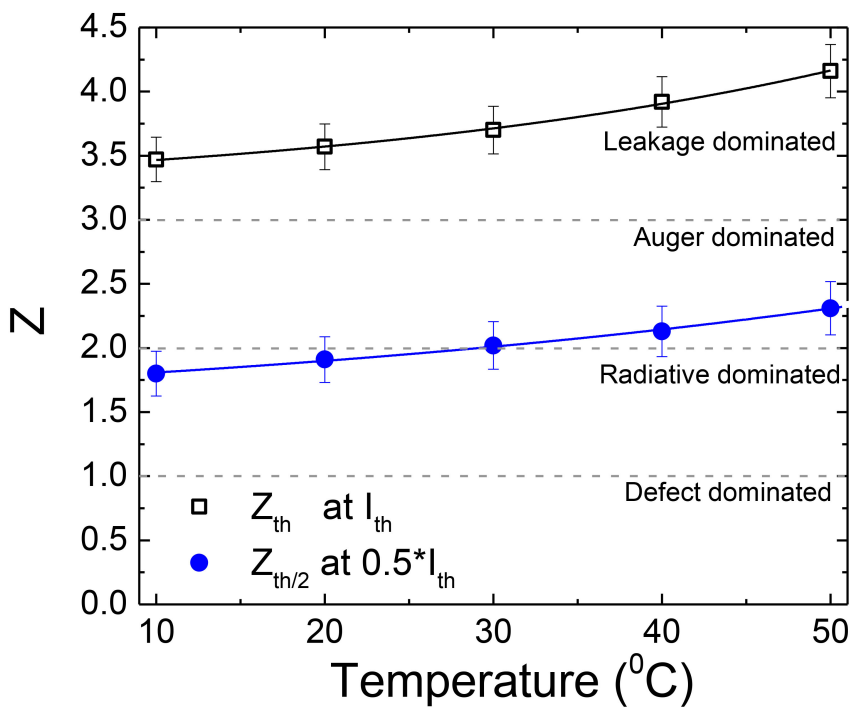

Fig. 5. $\quad \mathrm{Z}$ at threshold and half threshold as a function of temperature for the hybrid AlGaInAs/InP silicon-evanescent laser. Also marked are the theoretical values for Auger, radiative and defect dominated devices.

is known to be a problem in InP-based telecommunications wavelength lasers, where InGaAs and InGaAlAs based devices are found to have Auger contributions of as much as $~ 80 \%$ and $\sim 60 \%$ of the threshold current, respectively at room temperature. [33]-[35]. However, since $\mathrm{Z}$ increases above 3, this suggests that carrier leakage also plays a role at elevated temperatures, consistent with the increased thermal energy of the carriers and high injection levels. These mechanisms increase the temperature sensitivity of the lasers, as well as increasing the threshold current and lowering overall efficiency [36]. In addition, the low injection $\mathrm{Z}$ value of less than 2 (see eq.(1)) below $\sim 35^{\circ} \mathrm{C}$ is indicative of the presence of defects, possibly related to the bonding process.

Thus, the hybrid approach shows promise for the development of multiple self-aligned lasers on a silicon substrate. Clearly, however, factors limiting conventional InP-based lasers, such as Auger recombination and carrier leakage continue to be important in such devices and persist on the silicon platform. Additional non-radiative defect-related recombination, however, may be expected to reduce as the wafer bonding process improves.

\section{Direct Growth of III-V Active Regions ON SiLICON}

Direct epitaxial growth of III-Vs represents an idealised solution to III-V integration on $\mathrm{Si}$ in terms of fabrication complexity, speed and reliability; however the issues mentioned in Section I make realising monolithic integration a challenge. The industry standard telecommunications wavelength material systems based on GaAs and InP exploited through hybrid integration cannot be directly deposited on silicon with sufficient material quality owing to the large lattice mis-match.

It has been shown [37] that III-V-Sb materials exhibit some promise in managing defect propagation from the III-V/Si interface. It is found that strain is mostly compensated through the generation of Lomer-Cottrell dislocations [38], which are 
immobile in the slip plane and so tend to propagate in the plane of the III-V/Si interface, generating an interfacial misfit array [39]. The result is an effective blocking of the propagation of further threading dislocations in the slip plane, allowing high crystalline quality material to be grown in subsequent layers.

The groups of Huffaker and Tournie have developed $\mathrm{GaSb}$ and GaInSb MQW lasers on Si demonstrating electrical injection lasing up to 77 and $318 \mathrm{~K}$ with threshold current densities of 2 and $2.8 \mathrm{kAcm}^{-2}$, respectively [23], [37]. One issue that may need addressing in both cases is the requirement of a $5^{\circ}$ and $4^{\circ}$ miscut from the (100) direction of the Si surface, outside the usual maximum $0.5^{\circ}$ required for CMOS compatibility. Nevertheless achieving lasing at $1550 \mathrm{~nm}$ through monolithic integration on $\mathrm{Si}$ is an impressive achievement suggesting there is promise for the realisation of an efficient electrical injection laser on Si through the use of III-V-Sbs. This remains the subject of ongoing research.

The use of SSL to inhibit defect propagation has been in common use for some time [40]. It has been demonstrated however, that 3D island like structures formed through processes such as Stranski-Krastanow growth exhibit a larger strain field than the SSLs [41]. The larger strain field increases the strength of the Peach-Koehler force [42], more effectively bending, and by extension annihilating threading dislocations propagating in the slip plane.

Threading dislocations can be problematic in quantum well structures owing to the fact that carriers with high in-plane mobility will be trapped at the resulting defects and recombine non-radiatively. In quantum dot systems, this is less problematic owing to the fact that the carriers occupy spatially separated gain regions meaning that while a dislocation may inhibit gain in a given quantum dot, it will not affect the gain in the other quantum dots.

The use of defect blocking or filtering layers, as mentioned above has led to the rapid development of III-V quantum dots based lasers on Si. The work of Liu et al at UCL, for example, has recently demonstrated electrical injection $1.3-\mu \mathrm{m}$ InAs/GaAs quantum dot lasers on $\mathrm{Si}$ operating up to $111^{\circ} \mathrm{C}$ with a threshold current density of $200 \mathrm{Acm}^{-2}$ and output power of $100 \mathrm{~mW}$ at room temperature [43].

In addition, InAs quantum dots embedded in a GaAs/AlGaAs separate confinement heterostructure on $\mathrm{Si}$ have been developed by the group of Bowers and grown by MBE. These were found to lase under $\mathrm{CW}$ electrical injection at room temperature with a threshold current density of $\sim 500 \mathrm{Acm}^{-2}$. Thermal rollover limited the maximum operating temperature to $120^{\circ} \mathrm{C}$. Single facet wall plug efficiencies of $18 \%$, operation at $1.3 \mu \mathrm{m}$ and output power of $\sim 176 \mathrm{~mW}$ at ambient temperature indicate that these devices have potential for realisation of a viable silicon compatible laser [44].

An alternative approach investigated extensively at Philipps University, Marburg is the direct epitaxial growth of the dilute nitride GaNAsP on silicon using the more scalable MOVPE growth approach. Since GaP exhibits a very similar lattice constant to $\mathrm{Si}$, defect free growth of $\mathrm{GaP}$ on $\mathrm{Si}$ has been readily achievable and demonstrable for some time under the correct growth conditions [45]. GaP however is an indirect gap material



Fig. 6. Temperature dependence of $J_{\text {th }}$ in a GaNAsP/GaP SQW laser and its radiative component $J_{\mathrm{rad}}$ measured from integrated spontaneous emission and normalized in assumption that $J_{\mathrm{th}}=J_{\mathrm{rad}}$ at lowest T.

like $\mathrm{Si}$ and so is also unsuited to efficient photon generation. The addition of large As fractions forms a direct gap ternary, however the lattice constant mismatch is increased considerably. In order to form a direct gap material lattice matched to Si nitrogen in added to form the dilute nitride GaNAsP, which meets the aforementioned criteria for $\sim 4 \% \mathrm{~N}$ and $>70 \%$ As fractions [46]. In addition to this active material novel strain compensating boron containing materials have been developed to manage strain induced during growth by the differing thermal expansion coefficients. The materials $\mathrm{B}_{0.033} \mathrm{Ga}_{0.967} \mathrm{P}$ and $\left(\mathrm{B} \sim_{5 \%} \mathrm{Ga}\right)\left(\mathrm{As} \sim_{11 \%} \mathrm{P}\right)$ As are found to act as both suitable strain compensation layers as well as $\mathrm{SCH}$ and barriers respectively. Studies of the fundamental properties of these novel materials and their optimal growth conditions has been required in order to achieve defect free growth [47], [48].

Growth was carried out at Marburg by MOVPE, using an Aixtron AIX 200-GFR reactor with Pd-purified $\mathrm{H}_{2}$ as the carrier gas at a pressure of 50 mbar. The optimal reactor growth temperature was found to be $575^{\circ} \mathrm{C}$. Tertiarybutyl phosphine (TBP), tertiarybutyl arsine (TBAs), unsymmetric dimethyl hydrazine (UDMHy), triethyl gallium (TEGa) and triethyl boron (TEB) were used as sources for P, As, N, Ga and B, respectively. The contact layers were doped with diethyl zinc (DEZn) and diethyl tellurium (DETe) as sources for $\mathrm{Zn}$ and Te for $\mathrm{p}$ and n-dopants, respectively. More details, in particular about the MOVPE growth of these devices can be found elsewhere [49].

Initially devices were developed on $\mathrm{GaP}$ in order to optimise the structure and determine the efficiency limiting processes inherent to the material system before additional complications arising from the integration onto $\mathrm{Si}$ were introduced. Room temperature electrical injection lasing was reported with an emission wavelength of $981 \mathrm{~nm}$ and a threshold current density of $4 \mathrm{kAcm}^{-2}$ as shown in Fig. 6 [50]. Using the spontaneous emission technique discussed in Section III, by measuring the integrated spontaneous emission at laser threshold as a function of temperature the radiative component of the threshold current may be determined (open circles in Fig. 6). From this, it can 


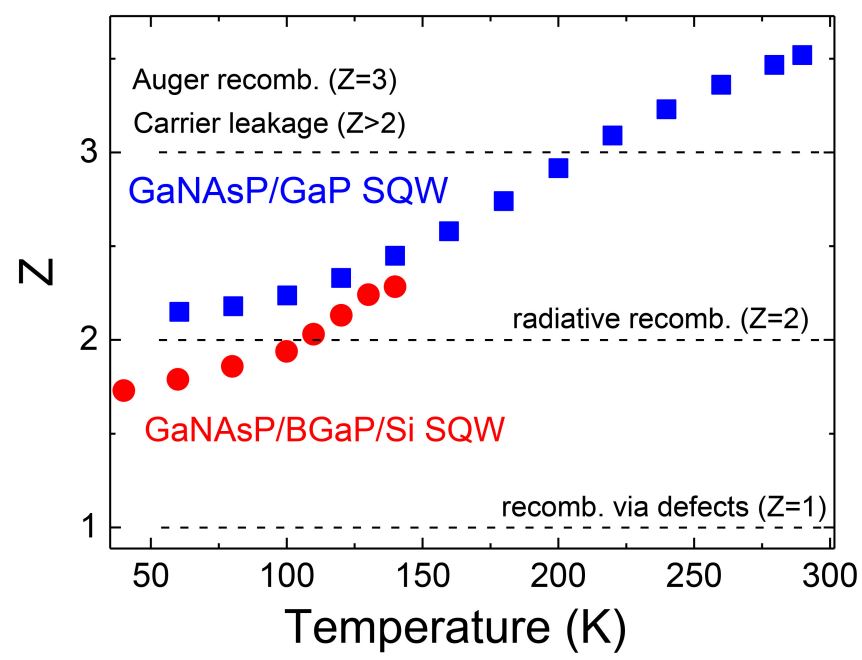

Fig. 7. Measured values of $\mathrm{Z}$ in a small current range below lasing threshold as a function of temperature in $\mathrm{GaNAsP} / \mathrm{BGaP} / \mathrm{Si} \mathrm{SQW}$ and $\mathrm{GaNAsP} / \mathrm{GaP} \mathrm{SQW}$ lasers.

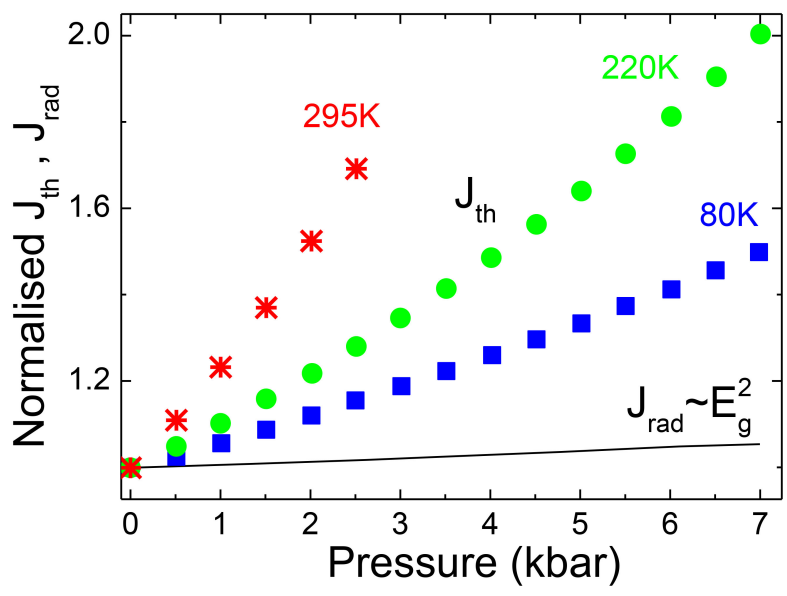

Fig. 8. Symbols show experimental normalized threshold current densities as a function of pressure at 80, 220 and $295 \mathrm{~K}$ in GaNAsP/GaP SQW laser. The radiative current (solid line) is obtained from the measured lasing wavelength assuming that $J_{\mathrm{rad}} \propto E_{g}^{2}$ as per conventional III-V based lasers.

be seen that radiative recombination accounts for $\sim 10 \% J_{\text {th }}$ at room temperature with the remaining $\sim 90 \%$ due to nonradiative recombination.

Fig. 7 shows the corresponding Z-analysis on the GaP-based devices (blue squares) from which a $Z>3$ is observed at the highest temperatures suggesting that carrier escape followed by non-radiative recombination plays a role (we note that Auger recombination is not significant at these wavelengths).

Further evidence for this may be seen in Fig. 8, which shows the hydrostatic pressure dependence of the threshold current where the threshold current is found to increase with pressure. This is consistent with increased carrier escape as pressure is applied consistent with the conduction band moving closer to the states into which the carriers escape. Since the structure contains several indirect band gap layers (at the $\mathrm{X}$-valleys) one might expect this to play a role. However, since this would cause a significantly stronger increase in $J_{\text {th }}$ with pressure, this

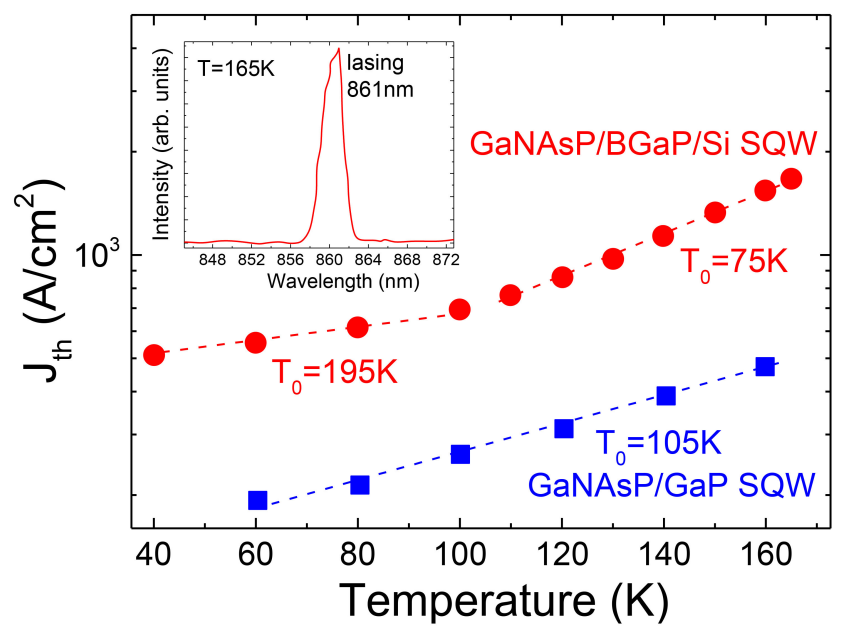

Fig. 9. Temperature dependence of $J_{\text {th }}$ in GaNAsP/BGaP/Si SQW and GaNAsP/GaP SQW lasers. Insert: lasing spectrum of the GaNAsP/BGaP/Si device at $165 \mathrm{~K}$.

is unlikely to be a dominant non-radiative path. In the absence of significant inter-valley carrier scattering, this suggests that carrier escape into defect-states outside of the quantum wells plays a role [51, 52].

Following the successful demonstration of devices grown on $\mathrm{GaP}$ substrates, devices were grown on $\mathrm{Si}$, with the first reports of a monolithically integrated III-V laser on Si being reported in 2011 by the Marburg group [53]. Electrical injection lasing took place up to a maximum temperature of $165 \mathrm{~K}$ with a threshold current density of $1.6 \mathrm{kAcm}^{-2}$ at a wavelength of $861 \mathrm{~nm}$ [54] as shown in Fig. 9. We note that the threshold current density of the Si-based devices (circles) is higher than that for the GaP-based devices (squares). There are many factors that may be responsible for this difference. To identify the dominant processes the Z-analysis and high hydrostatic pressure measurements proved to be very useful.

In Fig. 7 we also plot the temperature dependence of the Z-parameter for the silicon-based devices (red circles). Over the common temperature range for which data is available, we observe that $\mathrm{Z}$ is generally lower for the silicon-based devices decreasing below 2 at the lowest temperatures. This suggests that defect-related recombination in the active layer plays a more important role in these devices. This also suggests that through growth and design optimization such devices may expect to become at least as good as their GaP-based counterparts and show strong promise for this route towards silicon based lasers at room temperature.

\section{CONCLUSION}

In summary, the development of silicon based lasers has advanced significantly over the past few years driven by the appeal of full integration and convergence of electronics with photonics. In this paper we have shown that the integration of III-V based active materials on silicon remains one of the most promising approaches to achieve this aim owing to the inherent high optical transition strengths of III-V based semiconductors. Both 
wafer fusion and direct epitaxial approaches provide a practical and successful approach to achieving lasers on silicon. Experiments on such devices show in both cases that a reduction in non-radiative recombination can be expected to lead to further improvements in the scope and applicability of these innovative technological approaches.

\section{ACKNOWLEDGMENT}

The authors would like to express their deep gratitude and sincere appreciation to W. Stolz and K. Volz at Philipps University, Marburg and J. Bowers at UCSB for providing many of the state-of-the-art samples investigated in this study and for the many interesting and stimulating discussions on the topic of silicon based lasers.

\section{REFERENCES}

[1] Pew Research Centre, "Emerging nations embrace internet, mobile technology," Feb. 2014.

[2] R. S. Tucker, "Research at the university of Melbourne's centre for energyefficient telecommunications," IEEE Photon. Soc. News, vol. 27, no. 4, pp. 4-8, Aug. 2013.

[3] M. A. Taubenblatt, "Optical interconnects for high-performance computing," J. Lightw. Technol., vol. 30, no. 4, pp. 448-457, Feb. 2013.

[4] C. Duan and B. LaMeres, On and Off-Chip Crosstalk Avoidance in VLSI Design. New York, NY, USA: Springer Sci. Business Media, 2010.

[5] D. J. Blumenthal et al., "Integrated photonics for low-power packet networking," IEEE J. Sel. Topics Quantum Mech., vol. 17, no. 2, pp. 458-471, Mar./Apr. 2011

[6] D. J. Thomson et al., "50-Gb/s silicon optical modulator," IEEE Photon. Tech. Lett., vol. 24, no. 4, pp. 234-236, Feb. 2012.

[7] M. C. Tien, "Silicon photonic devices for optoelectronic integrated circuits," Ph.D. Ddissertation, Dept. Elect.rical Engineering. and Computer. Sciences., Los AngelesUniversity of. California at Berkley, Berkeley, CA, USA, 2009.

[8] H. Zappe, Fundamentals of Micro-Optics. Cambridge, U.K.: Cambridge Univ. Press, Sep. 2010.

[9] L. Pavesi, L. Dal Negro, C. Mazzoleni, G. Franzo, and F. Priolo, "Optical gain in silicon nanocrystals," Nature, vol. 408, pp. 440-444, Nov. 2000.

[10] A. G. Cullis and L. T. Canham, "Visible light emission due to quantum size effects in highly porous crystalline silicon," Nature, vol. 353, pp. 335-338, Sep. 1991.

[11] A. G. Nassiopoulos, S. Grigoropoulos, and D. Papadimitriou, "Electroluminescent device based on silicon nanopillars," Appl. Phys. Lett., vol. 69, pp. 2267-2269, 1996.

[12] D. Liang and J. E. Bowers, "Recent progress in lasers on silicon," Nat. Photon., vol. 4, no. 8, pp. 511-517, Aug. 2010.

[13] P. M. Fauchet, "Silicon light emitters: Preparation, properties, limitations, and integration with microelectronic circuitry in frontiers of nanooptoelectronic systems," NATO Sci. Series, vol. 6, pp. 99-119, 2000.

[14] O. Bisi, S. Ossicini, and L. Pavesi, "Porous silicon: A quantum sponge structure for silicon based optoelectronics," Surf. Sci. Rep., vol. 38, pp. $1-126,2000$.

[15] L. T. Canham, "Nanostructured silicon as an active optoelectronic material in frontiers of nano-optoelectronic systems," NATO Sci. Series, vol. 6, pp. 85-98, 2000.

[16] M. Dagenais, Integrated Optoelectronics. San Francisco, CA, USA: Academic, Oct. 2013.

[17] Y. De Koninck, G. Roelkens, and R. Baets, "Electrically pumped $1550 \mathrm{~nm}$ single mode III-V-on-silicon laser with resonant grating cavity mirrors," Laser Photon. Rev., vol. 9, no. 2, pp. L6-L10, Mar. 2015.

[18] M. H. T. Dastjerdi, A. Sanz-Velasco, J. Vukusic, M. Sadeghi, and J. Stake, "Transfer of InP-based HBV epitaxy onto borosilicate glass substrate by anodic bonding," Electron. Lett., vol. 46, no. 14, pp. 1013-1014, Jul. 2010.

[19] J. Sasaki et al., "Multiple-chip precise self-aligned assembly for hybrid integrated optical modules using AI-SN solder bumps," IEEE Trans. Adv. Packag., vol. 24, no. 4, pp. 569-575, Nov. 2001.

[20] J. Justice1 et al., "Wafer-scale integration of group III-V lasers on silicon using transfer printing of epitaxial layers," Nature Photon., vol. 6, pp. 610-614, Aug. 2012.
[21] D. Pasquariello and K. Hjort, "Plasma-assisted InP-to Si low temperature wafer bonding," IEEE J. Select Topics Quantum Electron., vol. 8, no. 1, pp. 118-131, Jan. 2002.

[22] B. Kunert, K. Volz, and W. Stolz, "Advances in the growth of latticematched III-V compounds on Si for optoelectronics," in Proc. 22nd Int Conf. Indium Phosphide Related Mater., 2010, pp. 1-4.

[23] A. Jallipalli et al., "1.54 $\mu \mathrm{m} \mathrm{GaSb/AlGaSb}$ multi-quantum-well monolithic laser at $77 \mathrm{~K}$ grown on miscut Si substrate using interfacial misfit arrays," Electron. Lett., vol. 43, no. 22, pp. 1198-1199, Oct. 2007.

[24] Z. Mi et al., "High-performance quantum dot lasers and integrated optoelectronics on Si," Proc. IEEE, vol. 97, no. 7, pp. 1239-1249, Jul. 2009.

[25] M. Groenert, C. Leitz, A. Pitera, and V. Yang, "Monolithic integration of room-temperature $\mathrm{cw} \mathrm{GaAs/AlGaAs} \mathrm{lasers} \mathrm{on} \mathrm{Si}$ substrates via relaxed graded GeSi buffer layers," J. Appl. Phys., vol. 93, no. 1, pp. 362-367, Jan. 2003.

[26] K. Samonji, "Reduction of threading dislocation density in InP-on-Si heteroepitaxy with strained short-period superlattices," Appl. Phys. Lett., vol. 69, pp. 100-102, 1996.

[27] D. Zhu, D. J. Wallis, and C. J. Humphreys, "Prospects of III-nitride optoelectronics grown on Si," Rep. Prog. Phys., vol. 76, pp. 1-31, Oct. 2013.

[28] Plessey Semiconductors Ltd. (2015). [Online]. Available: http://www. plesseysemi.com.

[29] R. Kumar et al., "An ultra-small, low-power, all-optical flip-flop memory on a silicon chip," Nature Photon., vol. 4, pp. 182-187, 2010.

[30] S. Srinivasan et al., "Hybrid silicon devices for energy-efficient optical transmitters," IEEE Micro, vol. 33, no. 1, pp. 22-31, Nov. 2012.

[31] A. Spott et al., "Heterogeneously integrated $2.0 \mu \mathrm{m} \mathrm{CW}$ hybrid silicon lasers at room temperature," Opt. Lett., vol. 40, no. 7 , pp. 1480-1483, 2015.

[32] A. Fang et al., "Electrically pumped hybrid AlGaInAs-silicon evanescent laser," Opt. Exp., vol. 14, no. 20, pp. 9203-9210, Oct. 2006.

[33] S. J. Sweeney et al., "Temperature and pressure dependence of recombination processes in $1.5 \mu \mathrm{m}$ InGaAlAs/InP-based quantum well lasers," Phys. Stat. Sol., vol. 241, no. 14, pp. 3391-3398, 2004.

[34] S. J. Sweeney, A. F. Phillips, A. R. Adams, E. P. O’Reilly, and P. J. A Thijs, "The effect of temperature dependent processes on the performance of 1.5 um compressively strained ingaas(p) MQW semiconductor diode lasers," IEEE Photon. Tech. Lett., vol. 10, no. 8, pp. 1076-1078, Aug. 1998.

[35] S. A. Sayid et al., "Thermal performance of $1.55 \mu \mathrm{m}$ InGaAlAs quantum well buried heterostructure lasers," in Proc. Indium Phosphide Related Mater. Conf., 2010, pp. 1-4.

[36] D. A. B. Miller, "Device requirements for optical interconnects to silicon chips," Proc. IEEE, vol. 97, no. 7, pp. 1166-1185, Jul. 2009.

[37] L. Cerutti, J. B. Rodriguez, and E. Tournie, "GaSb-based laser, monlithically grown on silicon substrate, emitting at $1.55 \mu \mathrm{m}$ at room temperature," IEEE Photon. Tech. Lett., vol. 22, no. 8, pp. 553-555, Apr. 2010.

[38] S. G. Balakrishan et al., "Growth mechanisms of highly mismatched AlSb on A Si substrate," Appl. Phys. Lett., vol. 86, pp. 1-3, 2005.

[39] S. Huang, G. Balakrishnan, and D. L. Huffaker, "Interfacial misfit array formation for GaSb growth on GaAs," J. Appl. Phys., vol. 105, no. 10, pp. $1-5$, May 2009.

[40] N. El-Masrya1 et al., "Defect reduction in GaAs epilayers on Si substrates using strained layer superlattices," Mater. Res. Soc. Meeting Proc., vol. 91, pp. 99-104, 1987.

[41] J. Yang, P. Bhattacharya, and Z. Mi, "High-performance $\operatorname{In}_{0.5} \mathrm{Ga}_{0.5}$ As/GaAs quantum-dot lasers on silicon with multiple-layer quantumdot dislocation filters," IEEE Trans. Electron. Devices, vol. 54, no. 11, pp. 2849-2855, Nov. 2007.

[42] M. Peach and J. Koehler, "The forces exerted on dislocations and the stress fields produced by them," Phys. Rev., vol. 80, pp. 436-439, 1950

[43] S. M. Chen et al., "1.3 $\mu \mathrm{m}$ InAs/GaAs quantum-dot laser monolithically grown on $\mathrm{Si}$ substrates operating over $100^{\circ} \mathrm{C}$," Electron. Lett., vol. 50, no. 20, pp. 1467-1468, 2014

[44] A. Y. Liu et al., "High performance continuous wave $1.3 \mu \mathrm{m}$ quantum dot lasers on silicon," Appl. Phys. Lett., vol. 104, pp. 1-4, 2014

[45] K. J. Bachmann, U. Rossow, N. Sukidi, H. Castleberry, and N. Dietz, "Heteroepitaxy of GaP on Si(100)," J. Vac. Sci. Technol. B, vol. 14, no. 4, pp. 3019-3029, Jul. 1996.

[46] B. Kunert, K. Volz, and W. Stolz, "Properties and laser applications of the GaP-Based (GaNAsP)-material system for integration to Si substrates in dilute III-V nitride semiconductors and material systems," Mater. Sci., vol. 105, pp. 317-341, 2008.

[47] N. Hossain et al., "Band structure properties of (BGa)P semiconductors for lattice matched integration on (001) silicon," in Proc. 31st Int. Conf. Phys. Semicond., pp. 47-48, Aug. 2012. 
[48] N. Hossain et al., "Band structure properties of novel BxGa12xP alloys for silicon integration," J. Appl. Phys., vol. 110, no. 6, pp. 1-5, 2011.

[49] S. Liebich et al., "MOVPE growth and characterization of Ga(NAsP) laser structures monolithically integrated on $\mathrm{Si}$ (001) substrates," in Proc. 22nd IEEE Int. Semicond. Laser Conf., 2010, pp. 143-144.

[50] N. Hossain et al., "Reduced threshold current dilute nitride $\mathrm{Ga}(\mathrm{NAsP}) / \mathrm{GaP}$ quantum well lasers grown by MOVPE," Electron. Lett., vol. 47, no. 16, pp. 931-933, Sep. 2011.

[51] N. Hossain et al., "Recombination and loss mechanisms in GaNAsP/GaP Qw lasers," in Proc. Photon. Global Conf., Dec. 2010, pp. 1-4.

[52] J. Chamings et al., "Temperature dependence and physical properties of $\mathrm{Ga}(\mathrm{NAsP}) / \mathrm{GaP}$ semiconductor lasers," Appl. Phys. Lett., vol. 93 , pp. 101-108, 2008.

[53] S. Liebich et al., "Laser operation of Ga(NAsP) lattice-matched to (001) silicon substrate," Appl. Phys. Lett., vol. 99, pp. 1-3, Aug. 2011.

[54] N. Hossain et al., "Physical properties of monolithically integrated $\mathrm{Ga}(\mathrm{NAsP}) /(\mathrm{BGa}) \mathrm{P} \mathrm{QW}$ lasers on silicon," in Proc. IEEE Int. Conf. Group IV Photon., 2011, pp. 148-150.



Graham William Read was born in England in 1988 He received the Masters degree in physics from the University of Manchester, Manchester, U.K., in 2011 Since 2011, he has been working toward the Ph.D degree in physics within the photonics group of the Advanced Technology Institute (ATI), University of Surrey, Surrey, U.K. His research has focused on the characterisation of III-V semiconductor lasers on silicon for use in optical interconnects. This has included investigation of lattice-matched novel dilute nitrides, hybrid wafer bonded devices and monolithic GaSb based lasers. His researched methodology includes novel temperature and pressure dependent characterization techniques for the identification of the physical processes which limit device efficiency.



Igor Pavlovich Marko was born in Belarus in 1974. He graduated with distinction from the Department of Quantum Radiophysics and Optoelectronics, Belarusian State University, Minsk, Belarus, in 1996. From 1996 until 2001, he studied the optical properties of $\mathrm{ZnSe}$ - and GaN-based wide bandgap semiconductors and optically pumped lasers at the Laboratory of Semiconductor Optics of B. I. Stepanov Institute of Physics of the Belarusian Academy of Sciences. He received the Ph.D. degree in 2000. Since 2001, he has been studying the properties of III-V based materials and photonic devices and systems from visible to far-infrared spectral range at the University of Surrey, Surrey, U.K



Nadir Hossain was born in Shariatpur, Bangladesh He received the B.Sc. (Hons.) degree from Khulna University, Khulna, Bangladesh, the M.Eng.Sc. (by research) degree from Multimedia University, Ayer Keroh, Malaysia, and the Ph.D. degree from the University of Surrey, Surrey, U.K. He is currently working as a Research Fellow at the INRS-EMT, Canada. Prior to joining INRS-EMT, he worked as a Research Fellow at the Advanced Technology Institute, University of Surrey. His key research interests are energy efficient novel photonic materials and devices for next generation "green" photonics technology. He was awarded NSERC PDF, UniS $\mathrm{PhD}$ Studentship in conjunction with Kwan Fellowship, Royal Academy of Engineering Fellowship, SPIE Education Scholarship in Optical Science and Engineering, IEEE and SPIE travel grants. His research work was selected as one of top 30 early career scientist's work (in physics category) in the U.K. to present at the House of Commons (SET for Britain). He has more than 100 scientific contributions which include referred journal papers, book/book chapters, full length conference articles, invited talks and conference presentations. $\mathrm{He}$ is the Member of IEEE Photonics Society, SPIE and the Optical Society of America.

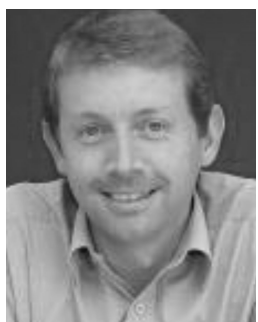

Stephen John Sweeney (S'98-M'99-SM'06) was born in England in 1973. He received the B.Sc. (Hons.) degree in applied physics and the Certificate in education from the University of Bath, Bath, U.K., in 1995, and the Ph.D. degree in semiconductor laser physics from the University of Surrey, Surrey, U.K., in 1999. After two years as a Postdoctoral Research Fellow working on InGaN LEDs and quantum dot lasers, he was Lead Scientist for Marconi optical components on the development of fixed frequency and tunable sources for communications applications. He is currently Full Professor of physics at the Advanced Technology Institute, University of Surrey, where he leads the photonics group. His current research interests include novel quantum well and quantum dot materials operating from the visible through to the mid-infrared for applications in solid-state lighting, lasers, detectors and photovoltaics. In addition to his role at Surrey, he is Chief Technology Officer for ZiNIR Ltd., a U.K.-based photonics SME, Associate Editor of IEEE JOURNAL OF QUANTUM ELECTRONICS and on the Editorial Board of the Journal of Materials Science: Materials in Electronics. He is a Member of SPIE, a Chartered Physicist and a Fellow of the Institute of Physics (London). 